Jahrbuch Schweiz - Dritte Welt 1995

\title{
Die Bedeutung neuer internationaler Vorstösse gegen die Korruption für die Schweiz
}

Mark Pieth

\section{(2) OpenEdition}

1 Journals

Electronic version

URL: http://journals.openedition.org/sjep/1284

DOI: $10.4000 /$ sjep. 1284

ISSN: 1663-9677

Publisher

Institut de hautes études internationales et du développement

Printed version

Date of publication: 1 février 1995

Number of pages: $254-261$

ISSN: 1660-5926

\section{Electronic reference}

Mark Pieth, « Die Bedeutung neuer internationaler Vorstösse gegen die Korruption für die Schweiz »,

Schweizerisches Jahrbuch für Entwicklungspolitik [Online], 14 | 1995, Online erschienen am: 05 Mai

2013, abgerufen am 08 September 2020. URL : http://journals.openedition.org/sjep/1284 ; DOI :

https://doi.org/10.4000/sjep.1284 


\title{
Die Bedeutung neuer internationaler Vorstösse gegen die Korruption für die Schweiz
}

\author{
Mark Pieth
}

\section{Korruption in der öffentlichen Diskussion}

Ein lange Zeit verdrängtes Problem ist zum Tagesthema geworden. Je genauer man hinsieht, um so verbreiteter erscheinen plötzlich korrupte Praktiken. Lange war die „kleine Korruption" des Alltags ein Standardthema von Reiseberichten aus der Dritten Welt. Sodann wurde von Industriellen, die mit Partnern in der Dritten Welt ins Geschäft kommen wollten, schulterzuckend darauf verwiesen, dass auch und gerade im Grossen ohne entsprechendes Handgeld kein Vertrag zu schliessen sei. Kurz: Korruption wurde als Problem der Dritten Welt definiert.

Trotz gelegentlicher „Affären“ auch in der industrialisierten Welt, ist die Erkenntnis neu, dass unter der Oberfläche auch in europäischen Staaten - und nicht nur in Italien - Korruptionskreisläufe von endemischen Ausmassen anzutreffen sind.

Korruption als Funktionsmechanismus einer Wirtschaft ist vollends ins Blickfeld gerückt mit der Öffnung der Märkte in Osteuropa, vor allem Russland. Auch wenn sogeünannte "Seilschaften“ von Funktionären bereits die Wirtschaft unter dem „ancien régime“ bestimmt hatten, erhält die Korruption nunmehr als Agens der Marktöffnung eine ganz andere Dimension. Die Parallelen reichen vom „wilden Westen“ bis zum „Manchester-Kapitalismus“. 


\section{Eingeschränkter Korruptionsbegriff}

Natürlich kümmert sich die öffentliche Diskussion - zumal in den Medien wenig um begriffliche Klarheit. Korruption steht für das unethische Geschäften schlechthin. Der Begriff dient auch als Chiffre, zur Schaffung neuer Feindbilder, die die diffusen Unsicherheiten und Verlustängste erträglicher machen sollen.

Definitionen, die das Problemfeld eingrenzen, offeriert primär das Strafrecht. Im Bereiche von Bestechung von Beamten enthält es regelmässig zwei Tatbestände, die eigentliche Bestechung (aktiv und passiv), nach der jemand einem Beamten eine Leistung dafür in Aussicht stellt, dass er dem ersteren in Verletzung seiner Amtspflicht einen jenem nicht zustehenden Dienst erweist (1). Geschädigt werden dabei möglicherweise andere Rechtssuchende, beeinträchtigt wird das Vertrauen in die Integrität der Beamtenschaft. Regelmässig enthalten die Strafgesetzbücher sodann die Variante der reinen Geschenkannahme, deren Schädlichkeit bereits in der abstrakten Gefährdung des öffentlichen Vertrauens in die Beamtenschaft gesehen wird (2). Demgegenüber enthalten die Gesetze zur Bekämpfung des unlauteren Wettbewerbs typischerweise ebenfalls strafbewährte Vorschriften gegen die Bestechung Privater hinter dem Rücken ihres Prinzipals (3). Zum Teil wird dieses Verhalten bereits durch den klassischen Straftatbestand der ungetreuen Geschäftsführung erfasst.

Eine Reduktion auf die strafrechtliche Perspektive würde allerdings die Diskussion erheblich einschränken: Das Strafrecht ist naturgemäss an der Zuschreibung von Verantwortung an Einzelpersonen interessiert, es hat Mühe damit, über die einzelne Tathandlung hinaus, die Langzeitstruktur und vor allem die Vernetzung der Korruption zu einem gesellschaftlichen und wirtschaftlichen Funktionsprinzip zu thematisieren. Erst recht gerät das Strafrecht bei der internationalen Dimension der "grossen Korruption" in Schwierigkeiten.

Es kommt aber nicht von ungefähr, dass bislang das Thema Korruption in der Schweizer Diskussion eingeschränkt auf die strafrechtliche Perspektive erörtert wurde. Sicher ist für die Schweiz und ihr Selbstverständnis bedeutsam, wie lange ein Raffael Huber ungehindert Bestechungsgelder fordern und entgegennehmen konnte, wie lange das System der Vermittlung von Bewilligungen im Gastgewerbe, trotz hartnäckiger Gerüchte, unentdeckt bleiben konnte. Allerdings handelt es sich - soviel wir wissen - nach wie vor um einen (zudem noch nicht rechtskräftig entschiedenen) Einzelfall aus einer Reihe von Einzelfällen, jedenfalls wenn wir den Blick auf die eigentliche Besteüchung einengen. Ausgeblendet wird in dieser Diskussion, dass die Bestechung nur ein Ausschnitt aus einem weiteren Thema, nämlich der Patronage und des Klientelismus (4) darstellt.

Während Bestechung sich in Geldwertvorteilen auszudrücken pflegt - und durchaus auch langjährige Beziehungen anstrebt, funktioniert sie aber aufgrund der täglichen Abrechnung in der entsprechenden Landeswährung. Demgegenüber vollzieht sich das Einfluss-Brokering über lange Zeit: Gefälligkeiten innerhalb eines "old-boys-network" sind über Jahrzehnte hinweg in Gegen- 
gefälligkeiten rückzahlbar.(Für Schweizer Parlamentarier wird dies keine neue Erkenntnis sein).

Besonders illustrativ für die selektive Pönalisierung von Klientelismus ist das Fehlen eines Parteienfinanzierungsgesetzes in der Schweiz. Bei einem erheblichen Teil der „Korruption“ in unseren Nachbarstaaten handelt es sich um unerlaubte Parteienfinanzierung. Der bewusste Verzicht auf eine entsprechende Regelung der Transparenz in der Finanzierung des politischen Prozesses vermeidet bereits auf dem Wege der Legalisierung des heimlichen "give and take" - zu Recht oder zu Unrecht mag hier offen bleiben - eine mögliche Form von Delinquenz. Der Verzicht auf Kriminalisierung wirkt wie eine Präventivmassnahme: Ein Musterbeispiel dafür, dass Kriminalität auch durch Zuschreibungsprozesse entsteht.

Durch Konzentration des Korruptionsbegriffes auf Bestechung und Bestechlichkeit im Sinne des StGB's wird die internationale Dimension grösstenteils ausgeblendet: Die entsprechenden Straftatbestände des StGB (insbesondere Art. 288, 315 und 316 StGB) schützen das Vertrauen der Bevölkerung in die Institutionen des (eigenen) Staates. Die Normen entstammen der Phase der Herausbildung der Nationalstaaten. Dass praktisch alle Nationalstaaten analoge Normen kennen, erlaubt allenfalls im Rechtshilfeverfahren, die Hürde der „beidseitigen Strafbarkeit“ zu überwinden, allerdings ist es aus Schweizer Sicht nach StGB nicht strafbar, Beamte eines ausländischen Staates zu bestechen. Hinzu kommt, dass nach bisheriger Praxis (5) in der Schweiz, wie in anderen Staaten, jedenfalls prinzipiell die internationale Bestechung im Steuerrecht als abzugsfähige Gewinnungskosten angesehen werden und damit indirekt Bestechung begünstigt wird.

\section{Internationale Korruption und die Schweiz}

Selbst wenn man von einem engen strafrechtlichen Begriff der Korruption ausgeht, ist zweifelhaft, ob die Ausgrenzung der internationalen Korruption heute noch adäquat ist. Die Schweiz wird grundsätzlich in dreifacher Weise von der internationalen Korruption berührt:

Als Exportland ist die Schweiz genauso wie andere Industrienationen an der aktiven Korruption ausländischer Beamter, insbesondere in der Dritten Welt beteiligt. Auch wenn einzelne Unternehmen die Grundsätze der internationalen Handelskammer (ICC(6)) beachten und weitere Unternehmen sich mindestens hausintern Richtlinien gegen die Korruption im Ausland auferlegen, sehen sich viele Exporteure zur Bezahlung von nicht-leistungsbezogenen Draufgeldern genötigt. Insofern nehmen Schweizer Unüternehmen - wenn auch meist unfreiwillig - an der Erschwerung des Entwicklungsprozesses in Ländern des „Südens“ und des "Ostens" teil.

Sodann kommt die Schweiz als "Tatort" in Frage: Liest man die Berichte etwa der Lockheed-Affaire, gehörte es zu den traditionellen Methoden, in der Schweiz ein Konto zu unterhalten, um die nötigen Bestechungszahlungen von 
hier aus abzuwickeln. Dieser Eindruck wird durch die italienischen Praktiken(7) bestätigt.

Zu denken ist aber auch an die „Bestechungsgeldwäscherei“ auf Schweizer Territorium: Manche Beamte suchen hier die erhaltenen Vorteile zu verbergen und zu legalisieren, dabei sind innen gelegentlich Schweizer Financiers behilflich.

\section{Internationale Vorstösse zur Eindämmung der Korruption}

Im Gefolge des Lockheed-Skandals, der weltweit bekanntgemacht hat, in welchem Ausmass nicht nur der US-Flugzeughersteller, sondern viele grosse multinationale Unternehmen, regelmässig höchste Regierungsbeamte des Auslandes bestachen, versuchte die UNO, eine Konvention gegen die internationale Korruption auszuarbeiten. Die Bemühungen, die von 1976 bis 1979 andauerten, scheiterten schliesslich an unüberbrückbaren Differenzen zwischen Industrienationen und Drittweltländern einerseits (im wesentlichen über die Einschätzung von Zahlungen an das Apartheidregime in Südafrika) und der Industriestaaten unter sich anderseits (über das US-Konzept der extraterritorialen Wirkung des Strafrechts (8).

Weitere Vorstösse der OECD $1976^{(9)}$ und der internationalen Handelskammer 1977 (10) blieben ohne tiefgreifende Wirkungen auf die internationale Korruptionspraxis.

1989 unternahmen die USA im Rahmen der OECD einen erneuten Vorstoss zur Erarbeitung eines internationalen Abkommens gegen „unerlaubte Zahlungen im internationalen Geschäftsverkehr". Dieser Intention mochten die OECD-Staaten nicht zu folgen, sie fanden sich aber bereit, eine Empfehlung auszuarbeiten, die am 27. Mai 1994 vom Rat der OECD verabschiedet wurde und inzwischen von den zuständigen Ministerien der Mitgliedstaaten mit Zustimmung bedacht worden ist.

Die Bedeutung dieses Textes liegt vor allem darin, dass die Länder der „Ersten Welt" sich bereit erklärt haben, alles zu unternehmen, um ihrerseits die aktive Korruption einzudämmen. Zu diesem Zweck haben sie einem „Followup-Verfahren“ in regelmässigen Abständen zugestimmt, das die Fortschritte in der Umsetzung der Richtlinien evaluieren und Vorschläge zur präziseren Fassung der Richtlinien vorbereiten wird. Vorerst soll damit vor allem die internationale Enttabuisierung einer Reihe von heiklen Themen erreicht werden, um die bisher ein grosser Bogen gemacht wurde: Insbesondere die steuerrechtliche Behandlung von Bestechungsgeldern und die Pönalisierung der Bestechung ausländischer Beamter.

Es versteht sich, dass Empfehlungen als sogenanntes „Soft-Law“ nur dann wirksam sind, wenn der gegenseitige Druck zur Umsetzung („peer pressure“) aufrechterhalten wird.

Während die Weltbank die Vergabe von Krediten regelmässig mit Bedingungen zur "Good Governance“ (etwa zur Demokratisierung, zur Verwaltungs- 
und Wirtschaftsreform) verbindet (11), soll die industrialisierte Welt mit dieser Richtlinie ihrerseits ihre Bereitschaft bekunden, gegen die aktive Korruption einzuschreiten. Es kann aber nicht übersehen werden, dass der Anlass zu diesem Schritt vorab das eigene Interesse der Wirtschaftsnationen an der Reduktion von Wettbewerbsverzerrungen war.

Ein wesentlicher Teil der üblichen „Good Governance“-Regeln besteht darin, Importrestriktionen und damit Machtbefugnisse und Ermessensspielräume einzelner Beamter abzubauen. Damit sollen die Gelegenheiten zur Bestechung reduziert werden, zugleich verfolgen die Industriestaaten damit ihr Interesse an der Öffnung der Märkte des „Südens". Es ist allerdings - sowohl bei der Umsetzung der OECD-Richtlinien, wie der "Good-Governance“-Regeln - zu beachten, dass Korruptionseindämmung nicht simple Deregulierung heissen kann. Vielmehr müssen spezifische Verwaltungsnormen, etwa im Bereiche des Buchhaltungs- oder aber des Kartellrechts, erst Bedingungen von Transparenz herstellen. Diesem Prinzip ist noch verstärkt Achtung zu verschaffen, wo Marktkräfte vorhanden sind, die ihre Ressourcen nicht aus legaler Konkurrenz beziehen müssen, die vielmehr über erhebliche „Kriegskassen“ aus der Untergrundwirtschaft verfügen. Zumal dort, wo Mittel des organisierten Verbrechens (insbesondere der Drogenmärkte) in den legalen Wirtschaftskreislauf drängen, ist Deregulierung nicht das alleinige Mittel der Wahl.

Inzwischen ist die Initiative der OECD aufgegriffen worden und von weiteren Organisationen, etwa der europäischen Justizministerkonferenz in Malta und dem Europarat, in einer eigenen Arbeitsgruppe weiterverfolgt worden.

\section{Schweizer Massnahmen gegen die Korruption}

Bereits Erwähnung gefunden haben die traditionellen Straftatbestände des Strafgesetzbuches gegen die aktive und passive Bestechung sowie deren Beschränkung auf die Bestechung Schweizer Beamter.

Eine Erweiterung hat das Abwehrarsenal mit Inkrafttreten des Gesetzes gegen das organisierte Verbrechen vom 1. August 1994 erfahren: Wie bekannt ist, gehören die Korruption und die Erpressung zu den bevorzugten Arbeitsinstrumenten krimineller Organisationen. Von besonderer Bedeutung ist es für die Schweiz, dass die Unterstützung einer solchen Organisation in ihrer kriminellen Tätigkeit neu bereits dann strafbar ist, wenn die Teilnahme oder Vorbereitung der eigentlichen kriminellen Haupttaten nicht nachweisbar ist (12).

Hinzu kommt, dass das neu gefasste Einziehungsrecht die erleichterte Abschöpfung von deliktisch erworbenen Vermögenswerten erlaubt. Insbesondere jene Werte, die in der Verfügungsmacht des organisierten Verbrechens stehen, sind separat und umfassend einziehbar(13). Wer der Unterstützung einer kriminellen Organisation überführt wird, muss in Zukunft obendrein dartun, dass seine Vermögenswerte nicht in der Verfügung der Organisation stehen, wenn sie nicht gesamthaft eingezogen werden sollen (diesbezüglich Umkehr der Beweislast) (14). 
Die Auswirkungen der neuen Geldwäschereistrafnorm Art. 305 bis StGB ist bei Bestechung schweizerischer Beamter geklärt: Die passive Bestechung ist ein Verbrechen, sie kann somit Vortat zur Geldwäscherei sein. Noch nicht ausgetragen ist indessen die Streitfrage, ob auch die Bestechung ausländischer Beamter als Vortat im Sinne der Geldwäschereinorm in Frage kommt (15). Jedenfalls stellt es nach neuester Praxis der Eidgenössischen Bankenkommission (EBK) einen Verstoss gegen das Bankaufsichtsrecht dar, vorsätzlich Bestechungsgelder von in- oder ausländischen Beamten anzunehmen (16).

Eine ganze Reihe von Regeln des Verwaltungs- und Zivilrechts dienen indirekt auch der Verhinderung der Korruption. Zu denken ist an Buchhaltungsvorschriften, an konkretisierende Bestimmungen, die das Ermessen zur Gewährung von Bewilligungen oder zur Verleihung von Aufträgen beschränken sollen, sowie die entsprechenden Kontrollvorschriften. Ebenfalls zur Vermeidung von Korruption im weiteren Sinne dienen Wählbarkeitsbeschränkungen in öffentliche Ämter, die dem Nepotismus Einhalt gebieten sollen, sowie entsprechende Ausstandsvorschriften.

\section{Die Aufnahme der internationalen Vorstösse in der Schweiz}

Etliche der angesprochenen Themen sind in der Schweiz bereits Gegenstand von Beratungen durch das Parlament und seiner Kommissionen. So wird derzeit von den Kommissionen für Wirtschaft und Abgaben (WAK) der Räte geprüft, wie die parlaümentarische Initiative Carobbio zur Abschaffung der steuerrechtlichen Abzugsfähigkeit von Bestechungsgeldern umgesetzt werden kann (17). Weitere Vorstösse sprechen die Ausdehnung der Strafbarkeit der aktiven Bestechung auf ausländische Beamte an (18).

Der Bundesrat hat seinerseits von den OECD-Empfehlungen in zustimmendem Sinne Kenntnis genommen und eine interdepartementale Arbeitsgruppe zur Prüfung von Wegen der Umsetzung eingesetzt. Vertreter der Wirtschaft zeigen sich insgesamt sehr interessiert an wirksamen Massnahmen zur Eindämmung der Korruption. Dem Prozess der OECD begegnen sie allerdings vorerst mit reserviertem Interesse. Das Gelingen von konkreten Schritten wird von der internationalen Beteiligung abhängen.

\section{Zusammenfassung}

Der Begriff Korruption steht für ein sehr weites Feld von moralisch und zum Teil auch rechtlich problematisierten Verhaltensweisen. Das Prinzip beruht auf elementaren Kommunikationsstrukturen und Überlebenstechniken, dem „do ut des“. Schädlich wird es dort, wo infolge von Intransparenz und ungleichen Zugangsbedingungen Martktverfälschungen bewirkt oder das Funktionieren von Behörden und Verfahren in Frage gestellt werden. Nur Ausschnitte des 
verbreiteten Klientelismus werden heute mit rechtlichen, zumal mit strafrechtlichen Mitteln bekämpft.

Die verbreitete Korruption ist für die Entwicklung der Dritten Welt besonders schädlich. Über die konkreten finanziellen Aufwendungen hinaus, die, auf den Kaufpreis überwälzt, vom Käuferstaat zu erbringen sind und inn noch stärker in die Abhängigkeit seiner Gläubiger bringen, stellt die verbreitete Korruption vor allem die Rechtsüberzeugungen und Ansätze zu demokratischen Strukturen in Frage.

Auch Unternehmen der schweizerischen Exportwirtschaft haben sich in der Vergangenheit an der Bestechung von Beamten im Ausland beteiligt. Die Schweiz, hat sich in verschiedenen Zusammenhängen als Tatort der Abwicklung internationaler Bestechungstransaktionen erwiesen, schliesslich ist die Bestechungsgeldwäscherei in der Schweiz beobachtet worden.

Im allgemeinen stimmt aber die Bereitschaft der offiziellen Organe sowie auch der Wirtschaft in der Schweiz, konkrete Schritte zur Eindämmung der Korruption zu unternehmen, optimistisch.

\section{Anmerkungen}

1. Vgl. Art. 288,315 StGB.

2. Beispiel für die Schweiz: Art. 316 StGB.

3. Beispiel für die Schweiz: Art. 4 a.u.b. UWG.

4. Vgl. Schmuel N. Eisenstadt/Louis Roninger, Patrons, Clients and Friends, Cambridge 1984 und Gioia Weber Pazmino, Klientelismus, Zürich 1991.

5. Vgl. dazu das Kreisschreiben der Eidg. Steuerverwaltung vom 8.11.1946.

6. Extortion and Bribery in Business Transactions, Report adopted by the 131 st. Session of the Council of the ICC, 29 November 1977.

7. Vgl. die Berichte zur "Pista Svizzera" der norditalienischen Bestechungsaffairen: Zum Beispiel Tagesanzeiger vom 22.9.1993 aber auch den Jahresbericht der Eidgenössischen Bankenkommission $1993 \mathrm{~S}$. $32 \mathrm{f}$.

8. Vgl. die Foreign Corrupt Practices Act von 1977, abgeändert durch die Omnibus Trade and Competitiveness Act 1988.

9. OECD-Richtlinie für Multinationale Unternehmungen vom 21. Juni 1976.

10. Vgl. oben Anm. 6.

11. So entwickelte die Weltbank 1993 bei der Vergabe eines Kredites an Kenia eine "Economic Governance: Anticorruption Policy Matrix" mit Problemkreisen, die die kenianische Regierung anzugehen hat.

12. Vgl. Art. 260 ter, i.Kr. seit 1. August 1994, dazu die Botschaft des Bundesrates, BBI 1993 III $277 \mathrm{ff}$.

13. Art. 59 Ziff. 3 StGB.

14. Ebda Satz 2.

15. Vgl. den unveröffentlichten Entscheid der Tessiner Rekurskammer vom 4.9.1992 S. 71 sowie Niklaus Schmid, Anwendungsfragen der Straftatbestände gegen die Geldwäscherei, vor allem StGB Art. 305 bis, in: Geldwäscherei und Sorgfaltspflicht, Schweizerischer Anwaltsverband 1991 S. $111 \mathrm{ff}$. und Paolo Bernasconi, die Bestechung von ausländischen Beamten nach schweizerischem Straf- und Rechtshilfe- 
recht zwischen EG-Recht und neuen Antikorruptions-Staatsverträgen, in ZStrR 109 (1992) S. $383 \mathrm{ff}$.

16. Jahresbericht der EBK 1993 S. 32.

17. Parlamentarische Initiative Carrobio vom 16. Juni 1993 (93.440).

18. Dringliche einfache Anfrage Rechsteiner vom 31. Mai 1994 (94.1059). 